\title{
Lack of association between Toxoplasma gondii exposure and depression in pregnant women: a case-control study
}

\author{
Cosme Alvarado-Esquivel ${ }^{1,5^{*}} \mathbb{B}$, Ana Liliana Martínez-Martínez ${ }^{1}$, Luis Francisco Sánchez-Anguiano², \\ Jesús Hernández-Tinoco², Juan Manuel Castillo-Orona', Carlos Salas-Martínez ${ }^{1,3}$, Antonio Sifuentes-Álvarez ${ }^{1,3}$, \\ Ada Agustina Sandoval-Carrillo², José M. Salas-Pacheco², Oliver Liesenfeld ${ }^{4,6}$ and Elizabeth Irasema Antuna-Salcido²
}

\begin{abstract}
Background: Very little is known about the link of T. gondii infection and depression. Through an age-, gender-, and month of pregnancy-matched case-control study, we determined the association of T. gondii infection and depression in pregnant women.

Methods: We studied 200 pregnant women with depression and 200 pregnant women without depression attended in a public hospital in Durango City, Mexico. Pregnant women were tested for the presence of antiToxoplasma lgG antibodies using an enzyme-linked immunoassay (EIA), and IgG seropositive women were further tested for the presence of IgM using an EIA. IgM positivity by EIA was further analyzed by enzyme-linked fluorescence assay (ELFA).

Results: Anti-T. gondii lgG antibodies were found in 9 (4.5\%) of the 200 cases and in 12 (6.0\%) of the 200 controls $(\mathrm{OR}=0.73 ; 95 \% \mathrm{Cl}$ : 0.30-1.79; $P=0.50)$. The frequency of high $(>150 \mathrm{lU} / \mathrm{ml})$ anti-T. gondii lgG levels was similar in cases and in controls $(\mathrm{OR}=1.20 ; 95 \% \mathrm{Cl}: 0.36-4.01 ; P=0.75)$. Two women were positive for IgM by ElA but both were negative by ELFA.
\end{abstract}

Conclusions: We did not find serological evidence of an association between T. gondii infection and depression in pregnant women attended in a public hospital in Durango City, Mexico. Since an association of T. gondii and depression in pregnancy has been reported in the U.S. previously, further research to elucidate the role of T. gondii in prenatal depression should be conducted.

Keywords: Toxoplasma gondii, Seroprevalence, Depression, Pregnant women, Case-control study

\section{Background}

Toxoplasma gondii (T. gondii) is a widely-distributed parasite [1], transmitted to humans by ingestion of raw or undercooked meat containing tissue cysts, and ingestion of food or water contaminated with oocysts shed by cats $[2,3]$. Primary infections in pregnant women may result in vertical transmission leading to congenital infections and disease $[4,5]$. Although most infections with T. gondii are asymptomatic, some infected individuals develop a

\footnotetext{
* Correspondence: alvaradocosme@yahoo.com

${ }^{1}$ Faculty of Medicine and Nutrition, Juárez University of Durango State,

Avenida Universidad S/N, 34000 Durango, Dgo, Mexico

${ }^{5}$ Laboratorio de Investigación Biomédica, Facultad de Medicina y Nutrición,

Avenida Universidad S/N, 34000 Durango, Dgo, Mexico

Full list of author information is available at the end of the article
}

disease called toxoplasmosis with involvement of eyes, lymph nodes and central nervous system $[6,7]$. Immunocompromised individuals infected with $T$. gondii are at risk for a reactivation of the infection leading to a severe disease mainly of the central nervous system [8]. Infection with $T$. gondii has been linked to psychiatric disorders including schizophrenia $[9,10]$, bipolar disorder, obsessivecompulsive disorder, and addiction [9]. However, the link between $T$. gondii infection and depression is controversial. In a Cuban study of psychiatric patients, those suffering from depressive disorders had the highest frequency of reactivity to the toxoplasmin intradermal test [11]. However, in a population-representative birth-cohort of individuals in Dunedin, New Zealand, T. gondii seropositivity 
was not significantly associated with major depression [12]. Similarly, in a meta-analysis of 50 studies into T. gondii infection for major psychiatric disorders versus healthy controls, no association between T. gondii IgG seroprevalence and major depression was found [9]. The association of T. gondii infection and depression in pregnant women has been poorly studied. Groër et al. found that higher anti-T. gondii IgG titers in infected women in the USA were related to depression and anxiety during pregnancy [13]. We aimed to determine whether $T$. gondii infection is associated with depression in pregnant women attended in a public hospital in Durango City, Mexico.

\section{Methods}

\section{Study design and population studied}

We performed an age-, gender-, and month of pregnancymatched case-control study of 200 pregnant women suffering from depression and 200 pregnant women without depression attended in a public hospital in Durango City, Mexico. This study was performed from March 2015 to February 2016. Inclusion criteria for enrollment of participants were: 1) pregnant women suffering from depression attending prenatal care consultations in the General Hospital of the Secretary of Health in Durango City; 2) aged 13 years and older; and 3) who accepted to participate in the study. Socioeconomic status was not a restrictive criterion for enrollment. Mean age in cases was $23.40 \pm 8.36$ (range 13-43) years old. Depressed pregnant women had 2-8 months of pregnancy (mean $6.5 \pm 1.5$ months). As a strategy to screen depression in pregnant women, validated Mexican versions of the Edinburgh postnatal depression scales (EPDS) (Additional file 1) were used in adults [14] and teenagers [15]. Pregnant women who screened positive for depression in the EPDS were further examined by a psychiatrist to confirm depression using the Diagnostic and Statistical Manual of Mental Disorders, Fifth edition criteria [http://www.dsm5.org/Pages/Defaul t.aspx]. Control pregnant women were matched with cases for age. Controls were randomly selected, and they scored negative for depression in the EPDS. Inclusion criteria for enrollment of control pregnant women were: 1) pregnant women without depression attending prenatal consultations in the General Hospital of the Secretary of Health in Durango City; 2) aged 13 years and older; and 3) who accepted to participate in the study. Mean age in control women was $23.01 \pm 7.55$ (range 13-45) years old. Pregnant women without depression had 2-9 months of pregnancy (mean $6.7 \pm 1.5$ months). No statistically significant differences in age $(P=0.62)$, and month of pregnancy between cases and controls were found.

\section{Detection of anti-T. gondii antibodies}

Serum samples from participants were obtained and stored at $-20{ }^{\circ} \mathrm{C}$ until analyzed. The presence of anti- $T$. gondii IgG antibodies was tested in sera using the commercially available enzyme immunoassay (EIA) kit "Toxoplasma IgG" (Diagnostic Automation/Cortez Diagnostics Inc., Woodland Hills, CA, USA). Anti-T. gondii IgG antibody levels were expressed as International Units (IU)/ml, and a cut-off for seropositivity of $8 \mathrm{IU} / \mathrm{ml}$ was used. Sera positive for anti-T. gondii IgG antibodies were further tested for anti-T. gondii IgM antibodies by using the commercially available EIA "Toxoplasma IgM" kit (Diagnostic Automation/Cortez Diagnostics Inc.). In addition, sera positive for anti- T. gondii IgM antibodies by EIA were further analyzed for these anti-T. gondii IgM antibodies using the commercially available enzymelinked fluorescent assay (ELFA) kit "VIDAS Toxo IgM" (BioMérieux, Marcy-l'Etoile, France). All IgG and IgM assays were performed following the instructions of the manufacturers.

\section{Statistical analysis}

Analysis was conducted using the software Epi Info 7 and SPSS 15.0 (SPSS Inc. Chicago, Illinois). For calculation of the sample size, we used a 95\% confidence level, a power of $80 \%$, a $1: 1$ proportion of cases and controls, a reference seroprevalence of $6.1 \%$ [16] as the expected frequency of exposure in controls, and an odds ratio of 2.8. The result of the sample size calculation was 195 cases and 195 controls. We used the student's $t$ test to compare the age among cases and controls. The association of T. gondii infection and depression in pregnant women was assessed with the two-tailed Pearson's chisquared test. Odds ratio (OR) and $95 \%$ confidence interval $(\mathrm{CI})$ were calculated, and a $P$ value $<0.05$ was considered statistically significant.

\section{Results}

Of the 200 cases of depression included in the study, $122(61.0 \%)$ suffered from minor depression, and 78 (39.0\%) from major depression. Anti-T. gondii IgG antibodies were found in $9(4.5 \%)$ of the 200 cases and in 12 (6.0\%) of the 200 controls. The seroprevalence of T. gondii infection was similar in cases and in controls $(\mathrm{OR}=$ 0.73; 95\% CI: $0.30-1.79 ; P=0.50$ ). Of the 9 anti- $T$. gondii IgG positive cases, $6(66.7 \%)$ had IgG levels higher than $150 \mathrm{IU} / \mathrm{ml}$, one (11.1\%) between 100 and $150 \mathrm{IU} / \mathrm{ml}$, and $2(22.2 \%)$ between 8 and $99 \mathrm{IU} / \mathrm{ml}$. In contrast, of the 12 anti-T. gondii IgG positive controls, 5 (41.7\%) had IgG levels higher than $150 \mathrm{IU} / \mathrm{ml}$, one $(8.3 \%)$ between 100 and $150 \mathrm{IU} / \mathrm{ml}$, and $6(50.0 \%)$ between 8 and $99 \mathrm{IU} / \mathrm{ml}$. The frequency of high $(>150 \mathrm{IU} / \mathrm{ml})$ anti-T. gondii IgG levels was similar in cases and in controls $(\mathrm{OR}=1.20$; 95\% CI: $0.36-4.01 ; P=0.75)$. Seroprevalence of $T$. gondii infection in patients with minor depression (4/122: $3.3 \%)$ was comparable to that $(5 / 78: 6.4 \%)$ found in patients with major depression $(P=0.29)$. Stratification by age 
groups (13-30 years, and older than 30 years) did not show differences $(P>0.05)$ in seroprevalences among cases and controls (3/149: $2.0 \%$ versus 10/159: $6.3 \%$, and 6/51: $11.8 \%$ versus $2 / 41: 4.9 \%$, respectively). Stratification by month of pregnancy groups ( $2-5$ months, and more than 5 months $)$ did not show differences $(P>0.05)$ in seroprevalences ( $2 \%$ versus $8.7 \%$, and $5.4 \%$ versus $5.2 \%$, respectively) or high IgG antibody levels ( $2 \%$ versus $4.3 \%$, and $3.4 \%$ versus $1.9 \%$, respectively) among cases and controls. None of the 9 anti-T. gondii IgG seropositive cases was reactive to anti-T. gondii IgM antibodies by EIA. Whereas 2 of the 12 anti-T. gondii IgG seropositive controls were reactive to IgM by EIA. These 2 IgM positive sera by EIA were negative to IgM by ELFA. Thus, none of the cases and controls was considered seropositive to IgM.

\section{Discussion}

Studies about the association of T. gondii infection and depression have shown conflicting results $[9,11,12]$. In addition, the association of $T$. gondii infection and prenatal depression has been poorly studied in particular. Therefore, the present study aimed to determine whether T. gondii infection is associated with depression in a sample of pregnant women in Durango City, Mexico. Results of tests for detection of $T$. gondii performed in the present study included qualitative and quantitative IgG and IgM assays. Our results do not point towards an increased rate of depression in pregnant women infected with $T$. gondii compared to matched control patients attended in the same hospital. We are aware of only one study on the link of $T$. gondii infection and depression in pregnancy. In such study, 414 women at 16-25 weeks of gestation in the USA were examined, and researchers found that higher $T$. gondii IgG antibody titers were associated with prenatal depression [13]. Authors hypothesized that immune escape of $T$. gondii may occur due to immune changes in pregnancy, and this could cause depression through activation of indoleamine 2, 3-dehydroxylase resulting in serotonin decrease [13]. It is not clear why the association of infection and depression was found in pregnant women in the USA but not in pregnant women in the current study. Comparison of both studies was based on IgG seropositivity; however, IgM seropositivity was not compared because this marker for acute or recent infection was not determined in the American study. It is possible that differences in the characteristics of pregnant women among the studies may explain the differences in the association. In the U.S. study, researchers examined women at 16-25 weeks of pregnancy whereas we examined women at 2-9 months of pregnancy. Stratification by month of pregnancy groups (2-5 months, and $>5$ months) did not show an association of infection and prenatal depression in the current study. While our study did not find any association of infection with $T$. gondii and depression in pregnant women several studies have demonstrated a link between $T$. gondii infection and depression. T. gondii seropositivity correlated with the Center for Epidemiologic Studies Depression score, Profile for Mood Statesdepression, and total mood disturbance score in a study of women veterans in the USA [17]. The age of women in the latter study was higher than in our study. Experimental evidence exists that reactivation of chronic $T$. gondii infection in mice by an immunosuppressive regimen caused depression-like behaviors, specifically, reduced sucrose preference, and increased immobility in the forced-swim test [18]. Researchers of the latter study also observed an enhanced tryptophan catabolic shunt and serotonin turnover that may be involved in the development of the depressive-like behaviors [18]. Reactivation of latent infection in humans is often observed in immunocompromised patients leading to life-threatening toxoplasmic encephalitis; it is therefore difficult to study an association with depression.

False positive results have been reported in anti-T. gondii IgM antibody tests [19]. Therefore, to increase the specificity of IgM seropositivity, we used two methods to test for anti- $T$. gondii IgM antibodies (EIA and ELFA). No acute cases of T. gondii infection were found, and therefore, treatment against $T$. gondii in the pregnant women studied was not needed.

Our study has limitations. First, we investigated the association of infection with $T$. gondii and depression in a relatively small cohort of pregnant women attending a public hospital. Therefore, our results cannot be extrapolated to pregnant women with different social status, i.e., those attended in private hospitals or other public hospitals. The great majority of women attended in the participating hospital had a low socioeconomic status.

\section{Conclusions}

We did not observe serological evidence of an association between $T$. gondii infection and depression in pregnant women attended in a public hospital in $\mathrm{Du}$ rango City, Mexico. Our results conflict with those reported in a previous study in the USA therefore warranting further research to elucidate the role of $T$. gondii in prenatal depression.

\section{Additional file}

Additional file 1: Tool used to screen depression. (DOCX $15 \mathrm{~kb}$ )

\section{Abbreviations}

Cl: Confidence interval; ElA: Enzyme-linked immunoassay; ELFA: Enzymelinked fluorescence assay; EPDS: Edinburg postnatal depression scale; IU: International units; ml: Milliliter; OR: Odds ratio; SPSS: Statistical package for the Social Sciences; USA: United States of America 


\section{Acknowledgement}

This study was financially supported by Juarez University of Durango State, Mexico. ALMM is a student of a master program with a grant of Juarez University of Durango State, Mexico. We thank study participants for their voluntary participation.

\section{Funding}

This study was financially supported by Juarez University of Durango State, Mexico.

\section{Authors' contributions}

CAE, LFSA, and JHT designed the study protocol, performed the data analysis and wrote the manuscript. ALMM, and JMCO obtained blood samples, submitted the questionnaires, and performed the data analysis. CSM and ASA performed the clinical assessment of participants. OL performed the data analysis, and wrote the manuscript. CAE, AASC, JMSP, and EIAS performed the laboratory tests. All authors read and approved the final version of the manuscript.

\section{Competing interests}

The authors declare that they have no competing interests.

\section{Consent for publication}

Not applicable.

\section{Ethics approval and consent to participate}

The Ethics Committee of the General Hospital of the Secretary of Health in Durango City, Mexico approved this study, and written informed consents were obtained from all participants and from the next of kin of minor participants.

\section{Author details}

${ }^{1}$ Faculty of Medicine and Nutrition, Juárez University of Durango State, Avenida Universidad S/N, 34000 Durango, Dgo, Mexico. ${ }^{2}$ Institute for Scientific Research "Dr. Roberto Rivera Damm", Juárez University of Durango State, Avenida Universidad S/N, 34000 Durango, Durango, Mexico. ${ }^{3}$ General Hospital, Secretary of Health, Avenida 5 de febrero 220, 34000 Durango, Mexico. ${ }^{4}$ Institute for Microbiology and Hygiene, Campus Benjamin Franklin, Charité Medical School, Hindenburgdamm 27, D-12203 Berlin, Germany. ${ }^{5}$ Laboratorio de Investigación Biomédica, Facultad de Medicina y Nutrición, Avenida Universidad S/N, 34000 Durango, Dgo, Mexico. ${ }^{6}$ Present Address: Medical and Scientific Affairs, Roche Molecular Systems, Pleasanton, CA 94588, USA.

Received: 12 June 2016 Accepted: 28 February 2017

Published online: 06 March 2017

\section{References}

1. Saadatnia G, Golkar M. A review on human toxoplasmosis. Scand J Infect Dis. 2012;44(11):805-14. doi:10.3109/00365548.2012.693197.

2. Jones JL, Dubey JP. Foodborne toxoplasmosis. Clin Infect Dis. 2012;55(6): 845-51. doi:10.1093/cid/cis508.

3. Montoya JG, Liesenfeld O. Toxoplasmosis. Lancet. 2004;363(9425):1965-76.

4. Kravetz J. Congenital toxoplasmosis. BMJ Clin Evid. 2013;2013:0906.

5. Torgerson PR, Mastroiacovo P. The global burden of congenital toxoplasmosis: a systematic review. Bull World Health Organ. 2013;91(7): 501-8. doi:10.2471/BLT.12.111732.

6. Harker KS, Ueno N, Lodoen MB. Toxoplasma gondii dissemination: a parasite's journey through the infected host. Parasite Immunol. 2015;37(3): 141-9. doi:10.1111/pim.12163.

7. Weiss LM, Dubey JP. Toxoplasmosis: a history of clinical observations. Int J Parasitol. 2009;39(8):895-901. doi:10.1016/j.jpara.2009.02.004.

8. Machala L, Kodym P, Malý M, Geleneky M, Beran O, Jilich D. Toxoplasmosis in immunocompromised patients. Epidemiol Mikrobiol Imunol. 2015;64(2):59-65.

9. Sutterland AL, Fond G, Kuin A, Koeter MW, Lutter R, van Gool T, Yolken R, Szoke A, Leboyer M, de Haan L. Beyond the association. Toxoplasma gondii in schizophrenia, bipolar disorder, and addiction: systematic review and metaanalysis. Acta Psychiatr Scand. 2015;132(3):161-79. doi:10.1111/acps.12423.

10. Alvarado-Esquivel C, Urbina-Álvarez JD, Estrada-Martínez S, Torres-Castorena A Molotla-de-León G, Liesenfeld O, Dubey JP. Toxoplasma gondii infection and schizophrenia: a case control study in a low Toxoplasma seroprevalence Mexican population. Parasitol Int. 2011:60(2):151-5. doi:10.1016/j.parint.2010.12.003.

11. Delgado García G, Rodríguez Perdomo E. Reactivity of toxoplasmin intradermal test in neurotic and manic-depressive patients. Rev Cubana Med Trop. 1980;32(1):35-9.

12. Sugden K, Moffitt TE, Pinto L, Poulton R, Williams BS, Caspi A. Is Toxoplasma gondii infection related to brain and behavior impairments in humans? Evidence from a population-representative birth cohort. PLoS One. 2016; 11(2):e0148435. doi:10.1371/journal.pone.0148435.

13. Groër MW, Yolken RH, Xiao JC, Beckstead JW, Fuchs D, Mohapatra SS, Seyfang A, Postolache $\Pi$. Prenatal depression and anxiety in Toxoplasma gondii-positive women. Am J Obstet Gynecol. 2011;204(5):433.e1-7. doi:10. 1016/j.ajog.2011.01.004.

14. Alvarado-Esquivel C, Sifuentes-Alvarez A, Salas-Martinez C. Validation of the Edinburgh postpartum depression scale in a population of adult pregnant women in Mexico. J Clin Med Res. 2014;6(5):374-8. doi:10.14740/ jocmr1883w.

15. Alvarado-Esquivel C, Sifuentes-Alvarez A, Salas-Martinez C. The use of the Edinburgh postpartum depression scale in a population of teenager pregnant women in Mexico: a validation study. Clin Pract Epidemiol Ment Health. 2014;10:129-32. doi:10.2174/1745017901410010129.

16. Alvarado-Esquivel C, Sifuentes-Alvarez A, Narro-Duarte SG, Estrada-Martínez S, Díaz-García JH, Liesenfeld O, Martínez-García SA, Canales-Molina A. Seroepidemiology of Toxoplasma gondii infection in pregnant women in a public hospital in northern Mexico. BMC Infect Dis. 2006;6:113.

17. Duffy AR, Beckie TM, Brenner LA, Beckstead JW, Seyfang A, Postolache TT, Groer MW. Relationship between Toxoplasma gondii and mood disturbance in women veterans. Mil Med. 2015;180(6):621-5. doi:10.7205/MILMED-D-14-00488.

18. Mahmoud ME, Ihara F, Fereig RM, Nishimura M, Nishikawa Y. Induction of depression-related behaviors by reactivation of chronic Toxoplasma gondi infection in mice. Behav Brain Res. 2016;298(Pt B):125-33. doi:10.1016/j.bbr. 2015.11.005

19. Liesenfeld O, Press C, Montoya JG, Gill R, Isaac-Renton JL, Hedman K, Remington JS. False-positive results in immunoglobulin M (IgM) Toxoplasma antibody tests and importance of confirmatory testing: the Platelia Toxo lgM test. J Clin Microbiol. 1997;35(1):174-8.

\section{Submit your next manuscript to BioMed Central and we will help you at every step:}

- We accept pre-submission inquiries

- Our selector tool helps you to find the most relevant journal

- We provide round the clock customer support

- Convenient online submission

- Thorough peer review

- Inclusion in PubMed and all major indexing services

- Maximum visibility for your research

Submit your manuscript at www.biomedcentral.com/submit
Biomed Central 